\title{
Against Brute Fundamentalism
}

\author{
Kerry McKenzIE \\ Penultimate draft of a paper forthcoming in dialectica. Please only cite the final version. \\ Copyright Kerry McKenzie.
}

\begin{abstract}
In metaphysics, the fundamental is standardly equated with that which has no explanation - with that which is, in other words, 'brute'. But this doctrine of brutalism is in tension with physicists' ambitions to not only describe but also explain why the fundamental is as it is. The tension would ease were science taken to be incapable of furnishing the sort of explanations that brutalism is concerned with, given that these are understood to be distinctively 'metaphysical' in character. But to assume this is to assume a sharp demarcation between physics and metaphysics that surely cannot be taken for granted.
\end{abstract}

This paper sets out to examine the standing of brutalism from the perspective of contemporary fundamental physics, together with theories of explanation drawn from philosophy of science and metaphysics. Focusing on what fundamental kinds the world instantiates and how physicists go about determining them, I argue that a partial explanation, in Hempel's sense, may be given of this fundamental feature. Moreover, since this partial explanation issues, at least in part, from stipulations as to the essential nature of the kinds involved, I claim that it has as much right to be regarded as a metaphysical explanation as do grounding explanations. As such, my conclusion will be that the doctrine of brutalism can no longer be regarded as tenable: at least modulo certain plausible essentialist assumptions, it is no longer the case that no explanation can be given of the fundamental.

\section{Introduction}

This paper addresses a certain metaphysical proclivity: that of reflexively equating the fundamental with that which has no explanation. It addresses, that is, our habit of identifying the fundamental with that which is simply brute. ${ }^{1}$ Consider for example the following quotes.

${ }^{1}$ This is the sense of 'brute' one typically finds in the literature when the term is articulated explicitly. Thus, for Fahrbach (2005, p. 449), 'Brute facts are facts that have no explanation'; for Barnes (1994, pp. 61, 62), they are those 'for which there is no further reason or explanation', those which occur 'for no reason whatsoever'. For Melnyk (1997, p. 636) brute facts are those that 'simply have no explanation'; for Parfit (1998b, p. 22), 'no explanation of any kind'. For Markosian (1998, p. 242), they are those for which we 'can just shrug and say, "There is no reason"'; for Dasgupta (2014, p. 9), those which are 'arbitrary', for which there is 'no rhyme or reason', and 'no explanation'. Similarly, G. Strawson (2006, p. 18) presents the 'brute' as that for which there is 'absolutely no reason in the nature of things' as to why it exists or is the way that it is. Now to be sure, sometimes 'brute' is taken in a stronger sense, a sense in which some explanation or reason may be given for brute entities, so long as those explanations fall short of entailing or determining them. Hudson (1997, p. 77), for example, in his discussion of the principle of sufficient reason, takes a brute fact to be one 'for which there is no sufficient reason or explanation' (italics added) as to why it holds. Since my argument concerns partial 
- 'Say that a fact is fundamental (or brute) iff it does not obtain in virtue of other facts' (Rosen 2012, p. 126).

- 'The fundamental (or brute) truths (if such there be) give us the ultimate foundation for the whole of reality' (Cameron 2015, p. 115).

- 'Fundamental or "brute" facts must be facts within physics' (Arno'dottir and Crane 2013, p. 252).

- 'There are those facts that are apt for having a ground but lack one. These are the so-called "fundamental” or "brute" facts (Dasgupta 2016, p. 7).

Such claims strike the contemporary metaphysical consciousness as perfectly unremarkable, and all present 'fundamental' and 'brute' as basically interchangable terms. It seems, then, that in metaphysics we are inured to the idea that being fundamental and having no explanation are essentially synonymous notions. As such, the idea that the 'fundamental facts just are those facts which have no further explanation' typically passes without any query or comment, much less with any objection. ${ }^{2}$ Call this idea that the fundamental entities lack any further explanation 'brutalist fundamentalism', or, for brevity, brutalism.

While the doctrine of brutalism has arguably been with us since antiquity, it finds renewed expression in much of the recent discussion on 'grounding'. Grounding has emerged as the prefered relation with which to relate the fundamental to the less fundamental, and so to define the 'layered' structure that Schaffer claims has moved to the fore. ${ }^{3}$ Such an approach motivates defining the fundamental in terms of its lack of ground. ${ }^{4}$ While debates abound as to how precisely one is to understand the notion of grounding, a common factor in all these discussions is that it is a non-causal explanatory relation of some sort. ${ }^{5}$ Indeed, grounding is often presented as the relation of 'metaphysical explanation', as though to give a metaphysical explanation just is to give a statement of grounds. Consider for example the following.

- 'I take grounding to be metaphysical explanation: to say that $\phi$ grounds $\psi$ is to say that $\phi$ provides a metaphysical explanation of $\psi^{\prime}$ (Litland 2013, p. 20).

- 'Some truths metaphysically explain, or ground, another truth just in case the laws of metaphysics determine the latter truth on the basis of the former' (Wilsch 2016, p. 1).

explanation - a form of explanation falling short of full determination - it will not touch, or at least not directly, on the brutalist's commitments if taken in this stronger sense. But since the weaker view in which the fundamental has 'no explanation whatsoever' seems to be that which is intended far more often, I both use the word with that meaning here and take others to do so similarly, unless they explicitly indicate otherwise. But in any case, my target is the canonical metaphysical thesis that the fundamental is that which has 'no explanation', however it is we choose to define the word 'brute'.

${ }^{2}$ DeRossett 2010, p. 81 (italics added).

${ }^{3}$ See e.g. Schaffer 2009.

${ }^{4}$ E.g. Schaffer op. cit., p. 373, takes ' $x$ is fundamental' to be defined by 'nothing grounds $x$ '. Of course, there are other approaches one could take to defining fundamentality. My argument will be largely independent of these debates: all that matters for my purposes is that what fundamental kinds the world admits constitutes a fundamental fact about it, however it is that 'fundamental' is ultimately defined.

${ }^{5}$ Some take grounding relations to be relations of explanation, others that which metaphysical explanations refer to. Either way, relations of grounding are understood to be essential to the provision of metaphysical explanations. 
- 'This attitude may be justified when it comes to some versions of the PSR... One way that this version differs from others is that it is concerned with one particular mode of explanation, what is nowadays referred to as metaphysical or grounding explanation." (Dasgupta 2016, p. 3)

All these statements present 'grounding explanation' as basically interchangeable with 'metaphysical explanation'. Thus if the fundamental is by definition that which is ungrounded, and if grounding is the relation of explanation relevant to metaphysics, then - as far as metaphysics is concerned at least - the fundamental is that which cannot be explained. But to define the fundamental in that way is just to express the doctrine of brutalism.

Brutalism, then, seems to get reflexive endorsement by metaphysicians, and is supported by a cluster of ideas circulating around the concept of grounding. However, for all its seeming innocuousness, I myself think we should be worried about brutalism. Prompting the worry is the fact that brutalism appears, on the face of it at least, to be in tension with another orthodox metaphysical doctrine - namely, the doctrine of physicalism. ${ }^{6}$ For physicalism is the thesis that 'the fundamental properties and facts are physical and everything else obtains in virtue of them'; as such, in worlds in which physicalism holds sway it is up to physics to discover what these fundamental facts and properties are. ${ }^{7}$ But while metaphysicians present these facts, whatever they may turn out to be, as that which by definition cannot be explained, it seems physicists have in recent years become increasingly optimistic about their ability to do just that. For Steven Weinberg, for example - Nobel Laureate and de facto chief spokesperson for the particle physics community - 'the aim of physics at its most fundamental level is not just to describe the world but to explain why it is the way it is' ${ }^{8}$ But it seems that by the lights of brutalism such an ambition is simply incoherent. And while we surely ought to maintain some degree of criticality over an utterance of one of particle physics' chief propagandists, I think we should be hesitant about attributing to him an incoherence in his attitude toward his subject matter.

There is of course an obvious response the brutalist might make to this worry, which is to point out that there need be no contradiction between their concept of the fundamental and the ambitions of physicists such as Weinberg. For while both physicists and metaphysicians are often acutely concerned with the fundamental, and indeed both in the business of providing explanations, the latter will claim that the sort of explanation that those scientists have in mind is not one that is relevant to their concerns. Indeed, everyone is happy with the idea that there are facts about the fundamental level that are open to causal explanation - such as why two electrons just collided where they did - so that the sort of explanation relevant to brutalism must be restricted to distinctively metaphysical forms if it is to be tenable. ${ }^{9}$ The fundamental's openness to scientific explanation thus does nothing to undermine the doctrine, and equally assuages any worries about burdening scientists with any conceptual incoherence. Regardless,

\footnotetext{
${ }^{6}$ In fact, this much not need be assumed for my purposes: all my argument requires is that the most fundamental physical facts are among the fundamental facts, even if they do not exhaust them. But seeing as physicalism 'is about as close to a bit of orthodoxy as one will find in contemporary philosophy' (Hall 2010), we might as well make the stronger assumption.

${ }^{7}$ Loewer 2001, p. 39.

${ }^{8}$ Weinberg 1992 (1994), p. 219.

${ }^{9}$ Cf. Dasgupta, op. cit, p. 6.
} 
then, of whether physicists succeed in their ambitions, the brutalist can relax. ${ }^{10}$

But for all that, I stand firm in my conviction that the time has come for us to give up brutalism. In what follows, I will make the case for that by (a) offering a model for how, in accordance with the aspirations of physicists, we might be said to explain some fundamental facts about the world (the more 'constructive' part), and (b) arguing that this puts pressure on the metaphysical doctrine of brutalism (the more 'critical' part). I will argue in particular that there exists a fundamental fact about the world - namely, the fact of what fundamental kinds it instantiates - for which we can provide a partial explanation in Hempel's sense, and that the partial explanation we obtain may justly be characterized as a metaphysical explanation. ${ }^{11}$ Hence it follows that we can no longer regard the fundamental as that which has 'no explanation', as the brutalist requires. In more detail, in Section 2, I say more about brutalism, emphasizing that according to it what fundamental kinds the world instantiates should be taken as something for which no explanation can be given. In Section 3, I make contact with fundamental physics by introducing quantum fields, and defend the stipulation that they are essentially such as to evolve unitarily. ${ }^{12}$ In Section 4, I argue that if we assume that the fundamental entities are quantum fields, so conceived, then what fundamental kinds the world can instantiate becomes subject to a significant constraint. In Section 5, I argue that this constraint amounts to a partial explanation of what fundamental kinds the world does instantiate, in the sense that Hempel understood the term, and moreover a partial explanation that deserves to be classified as metaphysical. In Section 6, I further defend that this is enough to undermine brutalism.

It will be clear, then, that the denial of brutalism I will argue for here will not come out of thin air. Rather, my partial explanation of the fundamental will take a swathe of facts for granted, some of which are surely controversial; indeed, since it will assume that the fundamental entities are quantum fields of some sort, it will take certain fundamental facts for granted. But note that there is nothing in this that undermines its status as an explanation. After all, I am not maintaining that nothing is brute, or that brute facts never appear in explanations, as would be required by the principle of sufficient reason; rather, my purpose is to dispute that the fundamental can be identified with that which has no explanation. Some fundamental features with some explanation would seem to be enough to establish that. Nor, as I will argue, is there anything about the fact that the explanans itself cites certain fundamental facts that 'trivializes' the explanation to be offered. For all that, the argument does have many moving parts, and I am sure that everyone will take issue with something. But I hope that we will at least be able to agree at the end that brutalism has been shown to be much less deserving a candidate for reflexive assent than has been previously thought. If the net result of the case to be laid out here is that brutalism becomes subject to the scrutiny befitting of a central metaphysical thesis - subsequently endorsed, if it is, in a sharpened-up form and for clearer and more conscious reasons - it will have served

\footnotetext{
${ }^{10}$ It should perhaps be noted that the assimilation of 'scientific' explanation with causal explanation specifically pervades both the metaphysics and the philosophy of science literature (see e.g. Loewer 2012 for a recent example). Recent work in philosophy of science has disputed this 'causal hegemony' (see e.g. Saatsi and Reutlinger forthcoming), and the present work implicitly disputes it too.

${ }^{11}$ Hempel's notion of 'partial explanation' will be described in Section 5 below.

${ }^{12}$ More on this meaning of this presently. But roughly, unitary evolution is what allows quantum superpositions to persist and for outcome probabilities to be consistent with the standard Born rule prescription for calculating them.
} 


\section{Tensions between Physicalism and Brutalism}

Brutalism, as noted, is the view that the fundamental is brute and inexplicable. Somewhat more fully, brutalism is the view that every fundamental fact, feature, or entity is wholly lacking in metaphysical explanation..$^{13}$ Thus were there a few facts about the fundamental that admitted of some metaphysical explanation - a few fundamental features here or there that we could explain in some measure - it seems that brutalism as a metaphysical doctrine would be thrown into crisis. Brutalism is, as it were, an all-or-nothing affair.

In worlds in which physicalism holds sway, as we have noted, the fundamental properties and fundamental facts are physical facts and properties. And among the fundamental physical facts about the world we may take to be facts concerning what fundamental kinds it instantiates. ${ }^{14}$ We do after all recognize a category of fundamental kinds, and we standardly take the world to instantiate some of them; we also take it to be physics' job to tell us which fundamental kinds these are. ${ }^{15}$ And which fundamental kinds the world does instantiate should be taken as a fundamental fact about it. ${ }^{16}$ For - to take an influential contemporary example - Sider takes fundamental kind terms such as 'electron' to be paradigms of terms that 'carve perfectly at the joints' ${ }^{17}$ As such, on this view, to correctly identify which fundamental kinds the world in fact instantiates just is to correctly specify a crucial aspect of the fundamental structure of the world. ${ }^{18}$ Thus if - as seems wholly uncontroversial - we take the fundamental structure of

\footnotetext{
${ }^{13}$ In what follows, I will talk of both facts and features as being fundamental. I will take the relata of explanations to properly be facts. Talk of explanations of events or features will be shorthand for talk of explanations for the fact that those events take place or that those features are instantiated.

${ }^{14}$ Here I will remain neutral on in virtue of what a kind should be classed 'fundamental': all we need to accept is that there are fundamental kinds, and that some are actualized.

${ }^{15}$ Cf. Paul 2012, pp. 5-6. For an attempt to cast scepticism on the idea that the world admits of fundamental kinds, see Schaffer 2003. Here however I stick with the orthodoxy by assuming some actual kinds are fundamental.

${ }^{16}$ Note that one need not take, as does Lowe (2006), the category of kinds to constitute a fundamental category in order to claim that facts about what fundamental kinds the world instantiates should be regarded as fundamental facts. (As Armstrong 1997, p. 67, puts it, 'The kinds mark true joints in nature. But it is not clear that we require an independent and irreducible category of universal to accommodate the kinds'.) For we can conceive of kinds as 'clusters' in some sense of perfectly natural properties, and take it to be a 'brute fact' which of these properties actually cluster so as to form the actual fundamental kinds (as does e.g. Chakravartty 2007, p. 171).

${ }^{17}$ Sider (2009, 2011 passim).

${ }^{18}$ Thus suppose, for now and for argument's sake, that the Standard Model of particle physics gives a true description of physical reality at its most fundamental (more on the status of this assumption momentarily). In that case, the up quark, the Higgs boson, and a massive electron neutrino will all count (along with others) among the world's fundamental kinds, so that distinctions between particles of these types will form (some of) the world's fundamental joints. It follows that at such time as we believed that protons and neutrons were fundamental - as opposed to mere conglomorations of quarks - then we were wrong about the fundamental structure of the world, since we mistakenly identified a non-fundamental joint with one of its fundamental joints. Further, at such time before anyone had mooted the existence of the Higgs, we were wrong about the world's fundamental structure on account of our failure to recognize a fundamental joint that it has. And at such time as we still believed that neutrinos were massless, we were also wrong about the fundamental structure of the world - this time because we attributed to it joints that it does not have, either fundamentally or otherwise.
} 
the world to be a fundamental feature of it, then which fundamental kinds the world instantiates should be taken as a fundamental feature of it too. And if - as also seems wholly uncontroversial - we take the fact that the world has a certain fundamental structure to be a fundamental fact about it, then the fact that the world instantiates whatever fundamental kinds it does instantiate should be regarded as a fundamental fact about it also. ${ }^{19}$

Let us then suppose - speaking somewhat more schematically now - that the fundamental kinds instantiated in the world are $\mathrm{K}_{1}, \ldots, \mathrm{K}_{\mathrm{p}}$, and let us collectively denote these by $\mathcal{F} \mathcal{K}$. Then just as the fact that the world has a certain fundamental structure is a fundamental fact about it, the fact that the fundamental kinds it instaniates are $\mathcal{F} \mathcal{K}$ is a fundamental fact about it also. Conjoining physicalism with brutalism then entails that the fact that the fundamental kinds instantiated by the world are $\mathcal{F} \mathcal{K}$ is a fact for which no metaphysical explanation may be given. Rather, the fact that the world is fundamentally carved up in this way is one that must simply be taken as brute.

A core commitment of physicalist brutalism, then, is that if the fundamental kinds the world instantiates turned out to be electrons, positrons, and photons, then there would be no explanation of why this is. Similarly, if the fundamental kinds the world instantiates turned out to consist of electrons and positrons but no photons, there would be no explanation of that either. And were the world's fundamental kinds to turn out to be electrons, photons, and a succession of 10 types of particle otherwise indistinguishable from electrons but for the fact each carries one unit of charge greater than the last, ending at charge 11e, then we would be at a loss to explain this as well; and so on. For concreteness, then, let us flag up this core implication of brutalist physicalism.

Brutalism about fundamental kinds $(B(\mathcal{F} \mathcal{K}))$ : Suppose that, of the fundamental kinds, those instantiated in the world are $\mathrm{K}_{1}, \ldots, \mathrm{K}_{\mathrm{p}}$. Let these kinds be collectively denoted $\mathcal{F} \mathcal{K}$. Then that the fundamental kinds that are instantiated in the world are $\mathcal{F} \mathcal{K}$ is a fact for which no metaphysical explanation can be given.

In what follows, I will take it as a datum that we are not, right now, in any position to claim to know what $\mathcal{F} \mathcal{K}$ is: that is, I will take it we are not at present in any position to assert what the actual inventory of fundamental kinds is. It is true that the most fundamental theory we have managed to produce to date, the Standard Model of particle physics, presents us with an inventory of kinds that we can with justification take to be more fundamental than any other that we know of, and of course we can list what these are. However, we have reason to think that that theory is not truly fundamental, and there are 'beyond the Standard Model' theories that posit more fundamental kinds still. But of course brutalism does not require that the Standard Model kinds are fundamental: all it requires is that whatever $\mathcal{F} \mathcal{K}$ should turn out to be, that it is the world's inventory of fundamental kinds is a fact that has no explanation.

As noted, however, while brutalism is very much the received view in metaphysics, it is a view that appears increasingly alienated from the perspective of fundamental physics. And what I want to claim here is that it is the physicists that are right on this score, because we can give that is, we can already give - at least a partial explanation of the fact that the fundamental kinds that are actually instantiated are the kinds $\mathcal{F} \mathcal{K}$. One might of course wonder how it is that we

${ }^{19}$ Cf. Sider p. 137. 
can say such a thing, given that we await knowledge of what $\mathcal{F} \mathcal{K}$ is: to ask for an explanation of $p$, after all, is typically to presuppose knowledge that $p$, and that is precisely what I claim we don't have. ${ }^{20}$ But what makes it possible to make this claim is that, while we may not know what the kinds featuring in $\mathcal{F} \mathcal{K}$ are, we can nevertheless derive a non-trivial constraint on them - something that, as we will see, supports the claim that we have a partial explanation of what fundamental kinds there are. And as we shall also see, what makes it possible to derive that constraint is that fact that we may be in a position to assert, even in advance of knowing the identities of the actual fundamental kinds, what sort of entity those kinds are all kinds of. String theorists, for example, will likely defend the claim that whatever the fundamental kinds are, they are all kinds of string. In what follows, however, and as noted in Section 1, we are going to assume that the fundamental kinds are all kinds of quantum field, and the time has come to say something about the motivation for and standing of that assumption.

This question of why we should make this assumption resolves itself into two: first, why make any assumption at all about what sort of physical entities the fundamental kinds are, and second, why take them to be quantum fields in particular. The first has an obvious (if rarely made explicit) answer, stemming from the fact that the sorts of entities that physics has presented us with, over the course of its history, have arguably differed profoundly from one another in ways that are metaphysically significant. ${ }^{21}$ As such, if we do not specify the sorts of entities that we take fundamental kinds to be, then paradigmatic metaphysical questions concerning them cannot be expected to have unambiguous answers. Nevertheless, the working assumption that those entities are quantum fields in particular surely warrants comment - not least because it may well be false, at least of the actual world. For the received view among physicists is that quantum field theory cannot incorporate gravity, and yet of course we know that gravity exists. Thus why assume that the fundamental kinds, whatever they may turn out to be, are all kinds of quantum fields? Why not assume that they are strings instead, given that string theory is widely taken as the most viable candidate for fundamental framework?

Several considerations support the present strategy. At the bottom of the list is the pragmatic justification that I simply do not know how else to proceed: QFT is the most fundamental framework both that we know how to test and that I myself have any facility with. Less pragmatically, however, we may say first of all that the fact that gravity exists is presumably contingent, and as such we can take it that there are possible worlds in which physicalism reigns and in which quantum fields are fundamental. Since brutalism is presented as a thesis about fundamentalia in general, hence intended as metaphysically necessary, if brutalism can be shown to be false in these worlds then brutalism is done for even if the actual world is not among them. ${ }^{22}$ Thus while for ease of presentation I will talk as though the fundamental kinds of the actual world are

\footnotetext{
${ }^{20}$ See e.g. Mellor 1976, p. 233: 'An explanandum is not an hypothesis, whose truth needs inferring from more surely known premises. It is already taken to be true, its truth indeed being what calls for explanation.'

${ }^{21}$ For example, quantum and classical systems have been argued to differ profoundly with regard to their status as individuals, whether they support holism, and whether they exhibit ontological vagueness.

${ }^{22}$ Note that Miller (2009) has disputed that 'propositions of metaphysics' are all necessarily true if true at all (as claimed by Sider 2013, p. 275). But her dispute does not extend to metaphysical propositions that are regarded as conceptual truths. Since we saw in Section 1 that 'being brute' and 'being fundamental' are presented as interchangeable notions, and hence (on the standard rendering) as synonymous notions, I take it that the brutalist regards their thesis as an example of a conceptual truth.
} 
all kinds of quantum field, should that turn out to be incorrect we can simply substitute throughout with a referring term for the appropriate worlds and still derive the sought conclusion. But furthermore - and more compellingly perhaps from a naturalistic point of view - the moves that will be made in what follows may be argued to be highly generic, and as such one can make a case that the central morals may be expected to carry over to whatever sorts of entities the actual fundamental kinds turn out to be. ${ }^{23}$ Finally, I should note that despite the seeming obstacles to incorporating gravity into the basic framework of QFT, there in fact exists an active programme attempting to do just that, and - while fledgling - this hypothesis is now enjoying some measure of success. ${ }^{24}$ If that programme does indeed succeed, then the conclusions drawn here regarding the explicability of the fundamental nature of the world may be more than just representative of how the correct argument will eventually go, but rather something more definitive. For all these reasons, then, I hope that despite the idealization it incurs with regard to the existence of gravity, there is reason to take the present argument against brutalism seriously.

With the (physical, metaphysical, and dialectical) justifications in place for assuming that the fundamental kinds are all kinds of quantum field, the claim I want to make is that once understood in this way then which fundamental kinds exist the world instantiates ceases to be a brute and inexplicable fact about it. Rather, it becomes a fact about the world that we can partially explain. In the next section, I will say a bit more about quantum fields and how I will interpret them, foregrounding the features that will be relevant to what is to follow.

\section{Introducing Quantum Fields}

To elucidate what is meant by quantum fields it is clearly to quantum field theory (QFT) that we must look, and we will begin with presenting the 'textbook theory' used in work-a-day physics (postponing for now any further interpretation). Put simply, QFT is the relativistic extension of quantum mechanics. As such, quantum fields are quantum systems evolving in Minkowski spacetime. To say that they are quantum systems is to say (i) that their states are capable of superposing and (ii) that the probability of obtaining a given term of the superposition upon a measurement is obtained via the Born rule. Thus supposing that $|\alpha\rangle$ and $|\beta\rangle$ are both possible states of a system, then (i) implies that $c_{1}|\alpha\rangle+c_{2}|\beta\rangle$ is likewise a possible state, and (ii) implies that the probability of finding the system in $|\alpha\rangle$ or $|\beta\rangle$ is given by $\left|c_{1}\right|^{2}$ and $\left|c_{2}\right|^{2}$ respectively. The basic problem that QFT addresses is how to characterize systems with this property compatibly with the structure of Minkowski spacetime, and a natural solution is to conceptualize quantum systems as operator-valued functions of it. Thus just as a classical field ascribes to each point of spacetime a real number (or n-tuple), a quantum field ascribes to each point a set of operators,

\footnotetext{
${ }^{23}$ This is because the present argument is based on the existence of constraints that issue from the requirement of mathematical consistency in the limit that takes us to the fundamental degrees of freedom. While what precisely these degrees of freedom are can be expected to differ across different physical frameworks, this is itself a generic requirement on fundamental theories that we can expect to be non-trivial in every case. (Unfortunately, in string theory is as yet unknown what these fundamental degrees of freedom are, including even whether they will involve energy or spacetime. As such, we are not in a position to talk about the constraints on fundamental string theories - meaning that it is not just my own lack of facility with the framework that accounts for why I have not framed the present discussion in terms of strings.)

${ }^{24}$ See Percacci 2009.
} 
the self-adjoint elements of which will correspond to observable quantities. ${ }^{25}$

One can show that a field defined in this way generically exhibits radical non-separability, in that in general the state of region $X \cup Y$ is not determined by the state in the subregions $X$ and $Y{ }^{26}$ Nonetheless, for all that QFT embeds a deeply holistic structure, the experimental signatures of particle physics concern well-localized phenomena, such as the discrete hits on a photomultiplier tube after a head-on particle collision. Thus approximately localized phenomena must be deriveable from quantum fields, and indeed they can be. ${ }^{27}$ As such, input and output quantum states $\left|\psi_{\text {in }}\right\rangle$ and $\left|\psi_{\text {out }}\right\rangle$, each featuring combinations of particle-like entities, may be defined in the theory. In a collision experiment, the particles in the input state will be directed so as to interact with one another, and what the possible experimental outcomes of this interaction are may be deduced via the $S$-matrix of the theory - something straightforwardly extracted from the Hamiltonian that describes the evolution of states from past to future times. ${ }^{28}$ Putting things schematically, then, we have

$$
\left|\psi_{\text {out }}\right\rangle=S\left|\psi_{\text {in }}\right\rangle .
$$

Now suppose that, as would be typical, our experiment consists of two protons $P$ with equal and opposite momenta of magnitude $|k|$ and energy $E$ colliding at some scattering centre. Such experiments are usually conducted with the aim of producing states of far greater richness than the generic input two-proton states. Thus assuming the energy is sufficiently high to pass the relevant particle mass thresholds, as a result of this experiment we will expect to find a number of different combinations of particles produced. Since many different combinations are generally possible consistent with the relevant conservation laws, the input state will be mapped by the dynamics to a superposition of possible outcomes, so that the outcome that we will observe will not in general be uniquely determined but rather expected with a probability given by the Born rule. Thus we have

$$
S|P(E, k) P(E,-k)\rangle=\sum_{i} c_{i}\left|T_{1}\left(k_{1}\right) \ldots T_{n}\left(k_{n}\right)\right\rangle .
$$

Here the $T_{j}$ denote different types of particle, so that the possible outcomes will in general feature many combinations of different particle types, and different numbers of each. ${ }^{29}$ (Note that here we could have $T_{i}=T_{j}$ for some $i, j$ : perhaps two photons with unequal momenta $k_{i}$ and $k_{j}$ are produced, for example.) Now let us simplify things and lump together all the outcomes featuring a given combination of particles, without worrying about how the energy-momentum is distributed among them, and let these lumped-together states be 'qualitative outcomes'. Thus putting things very schematically, we could, consistently with the various conservation laws, have the state of two protons evolve through interaction into one given by the sum of the follow-

\footnotetext{
${ }^{25}$ These operators will obey the canonical commutation relations and all other commutation relations that we take to be characteristic of a quantum system (for example, between the spin observables in different directions). To make the theory relativistic, we impose that the operators defined at spacelike separated points commute.

${ }^{26}$ See e.g. Wallace and Timpson 2010.

${ }^{27}$ At least in regions where measurements are made, far from the interaction centre: see e.g. Myrvold 2015.

${ }^{28} S$ is extracted from the Hamiltonian via $S=e^{i H t}$.

${ }^{29}$ The fact that particle number is not conserved is one of the innovations of relativistic quantum theory.
} 
ing qualitative outcomes:

$$
\left.\left.\left.S|P(k) P(-k)\rangle=c_{1} \mid 1 \text { heavy particle }\right\rangle+c_{2} \mid 3 \text { medium-mass particles }\right\rangle+c_{3} \mid 5 \text { light particles }\right\rangle
$$

In accordance with the usual prescription for relating states to probabilities of observation namely, the Born rule - the probability of observing the $i$ th of these qualitative outcomes upon a run of our accelerator is given by $\left|c_{i}\right|^{2}$. Since the idea that the probabilities associated with the elements of the sample space sum to one is an axiom of probability theory, the Born rule implies that we must have that $\sum_{i}\left|c_{i}\right|^{2}=1$ at all times. This condition that the sum of the $\left|c_{i}\right|^{2}$ 's must be preserved is encoded in the demand that the dynamics of quantum systems be norm-preserving. Together with the assumption that the dynamics be linear (in order to accomodate superposition), this means that the dynamics that features in textbook QFT is a unitary dynamics. ${ }^{30}$

What the textbook theory refers to as 'quantum fields', then, are systems in relativistic spacetime undergoing unitary evolution. In what follows, we will make the following strong - and strongly metaphysical - assumption: we will assume that quantum fields are not merely contingently but essentially such as to evolve unitarily. In other words, we will hold that unitary evolution is a necessary feature of quantum fields, true of them in every possible world in which they occur; though not merely in the sense that, for example, being such that $2+2=4$ is necessarily true of them, but rather in a way that is more reflective of their nature. ${ }^{31}$ This assumption of course warrants comment, with regard both to its standing vis-a-vis the interpretation of quantum theory and its implications for our metaphysics of physics.

Regarding the first, it should be noted that there exist interpretations of quantum mechanics in which unitarity is violated, such as the GRW collapse theory: while in that context the dynamics remains norm-preserving, it now contains non-linear collapse terms in addition to the linear Schrödinger evolution. However, the standing of collapse interpretations in relativistic settings is at present unclear at best. The only interpretation of quantum mechanics that is known to be consistent with the principles of relativity, namely the Everett (or 'many-worlds') interpretation, postulates only the dynamics of the 'textbook theory' and hence respects unitarity. As such, the only established candidate for an interpretation of QFT - a relativistic theory - in one in which fields evolve unitarily. While this is not the place to go into detail on the relationship of the many-worlds theory to modal theories more generally, suffice to say that our assumption of essential unitarity is at least consistent with the only interpretation of quantum theory that is known to be relativistically covariant. This surely counts as a point in its favour. ${ }^{32}$

\footnotetext{
${ }^{30}$ This postulate is expressed in the requirement that $S^{\dagger} S=1$, where ' $\uparrow$ ' denotes the Hermitian conjugate.

${ }^{31}$ See Fine 1995 . Note that it is compatible with this that their unitary evolution is a consequence of a more fundamental feature of quantum fields (hence part of their 'consequential' rather than their 'constitutive essence', in Fine's sense).

${ }^{32}$ It may be noted that the crucial constraints on particle content to be derived below rely explicitly (as we will see) only on the conservation of the norm, not linearity. Furthermore, the dynamics of the GRW theory is both norm-preserving and was designed, at least in the non-relativistic context, to reproduce the empirical predictions of quantum theory - and what particle content the world contains surely counts as one such. Nevertheless, it remains that the constraints that do the work in the ensuing argument were derived in the textbook theory, and it has not (to my knowledge) been established that these constraints on particle content are reproducible in frameworks that do not respect linearity. Hence for now I opt for safety over generality and focus on showing how the fundamental could be explained under one interpretation of quantum theory, postponing discussion of
} 
Regarding its status with respect to positions in the metaphysics of physics, the claim that quantum fields are essentially such as to evolve unitarily seems to constitute a quintessentially metaphysical assumption - one that has implications for the mathematical form of the dynamics in every possible world in which fields exist. As such, Humeans will likely reject it. But our aim here is not to generate an explanation of the fundamental that will be acceptable to everyone, only to show that an explanation of the fundamental is possible given some recognizable (and recognizably metaphysical) assumptions - for surely that suffices to undermine brutalism's status as an uncontroversial metaphysical doctrine. We can note further that in making this assumption we are at least in good company, since Ismael and Schaffer (2016) have recently expressed a similar conviction in their recent discussion of the metaphysics of the wavefunction. ${ }^{33}$ (The worry that there is something 'trivial' about the resulting explanation - an accusation often waged at anti-Humean explanation in general - will be addressed below in Section 6.)

Of course, much more could be said in defence of any claim that an entity is essentially such-and-such. Nevertheless, I take it that the assumption that quantum fields are not merely contingently but essentially such as to evolve unitarily constitutes a reasonable metaphysical assumption. With it in place, let us now see how we can put it use in explaining the fundamental.

\section{Pinning Down the Fundamental Kinds}

The task that we have set ourselves is to show that the fact that the world contains a certain suite of fundamental kinds, which we have denoted $\mathcal{F} \mathcal{K}$, is a fundamental fact about the world that may nevertheless be partially explained. As noted, one challenge we face in so doing stems from the fact that we do not know what the fields actually featuring in $\mathcal{F} \mathcal{K}$ are. But as has also been noted, there is a workaround here, predicated on the fact that we have postulated something about the natures of the systems involved - namely, that they are essentially such as to evolve unitarily. As we will see, this constrains $\mathcal{F} \mathcal{K}$ enormously, and this will go a long way to furnishing us with the claim sought. In this section we will focus on why it is that $\mathcal{F} \mathcal{K}$ is so constrained, bringing that constraint into contact with theories of explanation in the next section.

Our interest lies with the fundamental kinds, and among the laws that fundamental kinds accord with are of course the fundamental laws. ${ }^{34}$ Let us then take a moment to consider in virtue of what a law deserves to be called 'fundamental'. While one might try to capture this notion in a variety of ways, it seems uncontroversial to say that fundamental laws are those that operate at the very smallest spatial scales. ${ }^{35}$ Given that we are assuming that the fundamental entities

the strategy's generalization to collapse interpretations to another occasion.

${ }^{33}$ They write: 'There is a global constraint on the shape of the wave function, which is that its squared amplitudes must sum to 1. (Nothing else allows the wave function to play its calculational role with respect to the probabilities of observables. Since the amplitudes are squared to produce probabilities, a wave function whose shape violated this constraint would produce mathematical impossibilities.)... It might then be rejoined that [this] point about the wave (the global constraint that its squared amplitudes must sum to 1) represent[s] merely contingent happenstance.... We are skeptical of treating [this matter] as contingenc[y].'

${ }^{34}$ I say 'among' here because fundamental entities might be argued to accord with non-fundamental laws too. As such, we arguably cannot define a fundamental law simply as that which fundamental entities accord with.

${ }^{35}$ This is because non-fundamental laws in QFT may in principle be derived from more fundamental laws by the (irreversible) process of 'integrating out' high-energy degrees of freedom, hence the degrees of freedom describ- 
are housed in Minkowski spacetime, and that the latter has a continuous structure, it follows that fundamental laws of QFT must remain correct descriptions of phenomena at arbitrarily small scales. In this sense, the fundamental laws of QFT are required to be 'continuum compatible'. ${ }^{36}$ Since complementarity places an inverse relationship between energetic and spatial scales, it follows from this that a fundamental law of QFT is one that remains correct as a description even as interaction energies grow arbitrarily high. ${ }^{37} \mathrm{~A}$ fundamental law of quantum fields, then, is one that never needs supplanting no matter how fast our accelerators may grow, and hence one that continues to make correct empirical predictions even as energies get arbitrarily high. Now recall that quantum fields are here understood as systems whose nature is such as to undergo unitary evolution. Putting everything together, then, a fundamental law is one which, at a minimum, retains the property of being unitary even in the $E \rightarrow \infty$ limit.

This minimum necessary condition is easy enough to argue for, and even relatively intuitive. But while deriving this condition is straightforward, actually finding a law that satisfies it turns out to be extraordinarily difficult. The condition in fact turns out to be so demanding that prima facie we would not expect any given quantum field theory to succeed in meeting it. At the root of this difficulty lies the fact that, in QFT, the interaction couplings codifying the strength of interactions are themselves functions of energy, and one can show generically that if a coupling diverges in the limit then the calculated probabilities associated with the outcomes of the theory will diverge as well. ${ }^{38}$ One says in that case that the dynamics violates unitarity bounds, where such bounds are given by any mathematical relationship that follows from the requirement that the probability attached to any one empirical outcome can never exceed 1. Given the Born rule, it follows from this violation that at some energy the dynamics ceases to be unitary (since it is clearly no longer norm-preserving). The corresponding change of the strength of interaction couplings is described by the renormalization group equation associated with the theory, which describes the change for each interaction coupling $g_{i}$ in terms of a $\beta$-function:

$$
E \frac{\partial g_{i}(E)}{\partial E}=\beta\left(g_{i}, g_{j}, \ldots, E\right) .
$$

One can show that the $\beta$-function describing the change in the behaviour of any one coupling is a function of all the couplings $g_{j}$ that feature in the theory, and hence (it turns out) the spins and masses of all the fields present. ${ }^{39}$ This makes the act of actually solving any one of these equations a rather formidable mathematical challenge, and as such it is extremely difficult to show that the couplings will tend to a finite value in the limit, as required of a fundamental

ing processes in the smallest spatiotemporal regions (see e.g. Castellani 2002). Since the fields whose behaviour is described in these laws have - as noted above in Section 3 - a highly holistic character, the state-dependent properties that these regions instantiate will in general be a function of the properties of the whole. As such, there is scope to combine this conception of fundamental laws with a 'priority monism' at the level of properties (cf. Schaffer 2010): for in order for the laws to correctly describe the properties of the very smallest regions, they must also correctly describe the whole.

${ }^{36} \mathrm{Cf}$. Huggett and Weingard 1995. General considerations in quantum gravity suggest the possibility of a discrete spacetime. But that possibility remains speculative (even more so than the theories themselves).

${ }^{37} \mathrm{We}$ can take it as a given that the fundamental law is a law of interaction. It is hard to see how there could even be such a thing as a levels structure at all without interactions in the base.

${ }^{38}$ See Percacci 2012.

${ }^{39}$ See e.g. Collins 2008, Sec. 7.7. 
theory. Nevertheless, despite these mathematical challenges, it is not difficult to be convinced that actually finding a theory that stays norm-preserving in the limit is likely to prove extremely difficult. For consider that the probabilities $c_{i}$ attached to the qualitative outcomes in expression (3) above are in general going to be functions of the interaction energy. (For example, in order to create the Higgs particle, we had to wait until we had an accelerator that could supply an energy equal to its mass. But since the probability attached to the qualitative outcome containing that particle switches at that energy from zero to something finite, then the probabilities attached to the qualitative outcomes creatable at lower energies must correspondingly go down - probabilities must, after all, sum to one.) As such, it is clear that each $c_{i}=c_{i}(E)$. Now consider the probability functions that are implied by a fundamental theory in particular. Since the laws of this theory must be well-defined in the $E \rightarrow \infty$ limit, each $c_{i}$ must at the very least stay finite in that same limit - for otherwise the theory is not well-defined, and certainly the $\left|c_{i}\right|^{2}$ make no sense as probabilities. But a priori we know that it is only a very special class of functions the convergent functions - that stay finite as their variables diverge. In this sense, we know that the fundamental law must have a rather special mathematical structure to imply only functions of this sort - the set of convergent functions being a measure-zero set after all.

But this turns out to be just the tip of the iceberg when it comes to the mathematical 'specialness' of fundamental theories: for in order to stay well-defined in the limit not only must any one probability function be rather special individually, but they must also be supremely special collectively. For simple combinatorics, combined with the fact that energies can in principle increase without limit (at least according to QFT), means that there is equally no limit in principle to the number of possible combinations of particles that can be produced in interactions as energies grow high enough. Thus we can see that the number of qualitative outcomes associated with a fundamental theory will tend to infinity in the same limits in which it is required to be well-defined. And yet it must be that the $c_{i}$ associated with each of these outcomes remain so exquisitely in concert with one another that they continue to sum to one, no matter how high the energy grows, and hence no matter how many qualitative outcomes must be taken into consideration - even though we know that there is no limit in principle to how many this is. Hopefully no further persuasion is needed to grant that keeping so many balls in the air at once is a highly non-trivial mathematical feat, and hence that writing down a law that actually has this feature is far from straightforward. Thus even before we start thinking about whether or not a given candidate expression for interactions between quantum fields is 'correct' in the sense of empirically adequate, we are going to have our work cut out establishing that it is even a coherent possibility.

Given that continuum-compatible laws, then, are going to be extremely rare finds, one might wonder what the hallmarks of a theory are that suggest it is befitting of that accolade. Predictably, however, any attempt to address this question must confront the rather intimidating mathematical intractabilities gestured at above, and partly as a result of this we are as yet unable to say anything that is fully general. However, there exists a special class of fundamental theories for which we do have demonstrable and precise criteria. This is the class of theories whose interaction couplings, upon which probabilities depend, not only stay finite in the $E \rightarrow \infty$ limit - a condition known as asymptotic safety - but furthermore tend to zero. These latter theories are known as asymptotically free. It will be clear that the asymptotically free theories are but a special class of fundamental theories: there are, after all, many more ways to stay finite than to tend to zero in particular. Nevertheless, one can argue that something comparable to what 
is true for those will also be true of the more general class, in that in each case we can expect stringent constraints on field content although the precise nature of the constraints will likely be different in the two cases. ${ }^{40}$ As such, in what follows I will explicitly discuss only the case of asymptotically free theories, and use those as our model of fundamental theories; but it should be borne in mind that the precise nature of the constraints on kind content to be deduced here will likely be different in the general case.

What, then, are these constraints that asymptotically free theories - those serving as our model of fundamental theories - have to satisfy? The answer emerged across a series of seminal papers published in 1973. The first relevant result for our purposes was shown by Coleman and Gross, who argued, in a proof by cases, that only theories containing non-Abelian gauge bosons had a claim on being fundamental. ${ }^{41}$ In more detail, they argued that if a theory contains any Abelian gauge bosons, the $\beta$-function associated with the theory will diverge and hence unitarity bounds be violated; similarly if a theory contains only spinless mesons; and similarly if it contains only spinless mesons and fermions. (Since they were focussing on 'renormalizable' theories they did not explicitly consider theories containing only fermions, but it can be shown that non-renormalizable theories cannot be asymptotically free either. ${ }^{42}$ ) In all cases in which non-Abelian gauge bosons are absent, then, the interaction couplings will fail to approach zero in the $E \rightarrow \infty$ limit, leading them to conclude that it is impossible to achieve asymptotic freedom without the presence of non-Abelian gauge bosons. And although I will not do so here, it may be shown that the number of bosons in any given case is uniquely determined by the symmetry of the theory in question: for example, QCD - the theory of the strong interactions - will contain 8 gauge bosons ('gluons') as a result of the fact that it is based in the gauge group SU(3). ${ }^{43}$ It should be underlined that this is a significant constraint upon quantum field theories, as there are plenty of workable or 'effective' theories that nevertheless fail to feature gauge bosons: famous examples are the Fermi model of the weak interaction, or the Nambu-Jona-Lasiño model of condensed matter, both of which contain fermions alone. What Coleman and Gross' result tells us is that, while these may be perfectly good as non-fundamental descriptions of nature, they will nevertheless break down somewhere. As such, they cannot be candidates for fundamental descriptions.

The second relevant result, shown first by Gross and Wilczek, is that the presence in a gauge theory of too many fermions will have an effect on the $\beta$-function that tends to destablize

\footnotetext{
${ }^{40}$ The problem in both cases is that the renormalization group equation (4) that determines whether a theory is continuum compatible or not is a function of all the couplings in the theory. But in QFT masses function as a kind of coupling, and the dimension of a coupling is determined by the spins of the coupled fields. Thus for any fundamental theory to be well-behaved in the limit, there will have be constraints on its matter content, even if we do not have the wherewithal to determine precisely what these constraints are in every case. One might say that the underlying disease is the same in all instances, although the details of the treatment will differ.

${ }^{41}$ Coleman and Gross 1973 . While the mathematical vocabulary will not be defined here, suffice to say that nonAbelian gauge bosons are bosons associated with non-Abelian symmetry groups, and include particles such a gluons. Photons are an example of Abelian gauge bosons.

${ }^{42}$ Theories containing only fermions are always non-renormalizable, and asymptotically free theories are always renormalizable: see Weinberg 1979, p. 806.

${ }^{43}$ The set of gauge bosons is identified with the adjoint representation of the relevant symmetry group, and what this is is determined by the group structure itself.
} 
a theory's asymptotic freedom. ${ }^{44}$ As such, while some fermions are permitted, there is an upper limit on how many there can be. ${ }^{45}$ For example, Gross and Wilczek showed that QCD can accommodate up to 16 types of fermion, but any more will cause it to run afoul of asymptotic freedom. Again, it should be emphasized that what this number is is determined by the symmetry of the theory, and as such is intimately related to the number of gauge bosons that are present. Thus in QCD the fact that up to 16 fermions are allowed is closely related to the fact there are 8 bosons.

Putting everything together, then, we may say that a theory can be expected to be asymptotically free only if:

- the theory contains some number $\mathrm{N}>0$ of non-Abelian gauge bosons;

- the number of fermions does not exceed some number $\mathrm{M}$, with $\mathrm{M}$ intimately related to $\mathrm{N}$.

We seen, then, that theories cannot contain too many types of field if it is to exhibit asymptotic freedom, but they cannot contain too few either. It therefore seems right to say that if the fundamental entities are quantum fields - and hence the fundamental kinds are all kinds of quantum field - then a sort of 'Goldilock's principle' constrains the combinations of fundamental kinds that can co-exist at a world. Taking $\mathrm{B}_{\mathrm{i}}$ to be bosonic fields and $\mathrm{F}_{\mathrm{i}}$ fermionic, we may state this principle as follows:

Goldilock's principle for fundamental kinds $(G P)$ : Whatever the actual inventory of fundamental kinds $\mathcal{F} \mathcal{K}$ is, it will take the form $\mathrm{B}_{1}, \ldots \mathrm{B}_{\mathrm{N}} ; \mathrm{F}_{1} \ldots \mathrm{F}_{\mathrm{M}}$, for some $\mathrm{N}>0$ and with an upper bound on $\mathrm{M}$, and with $\mathrm{M}$ and $\mathrm{N}$ related.

The $G P$ is clearly a significant constraint on a law of nature: it is emphatically not the case that an arbitrarily selected combination of fields will be expected to satisfy it. Thus we cannot expect an arbitrarily selected list of fundamental fields to be a candidate for $\mathcal{F} \mathcal{K}$. Nevertheless, for all that it represents a highly non-trivial constraint, it is crucial to note that the $G P$ does not itself pin down $\mathcal{F} \mathcal{K}$ uniquely. Not only does it fail to determine precisely which bosons are realized (while severely constraining what the possibilities are), but there is also some tolerance in how many types of fermions are permitted. In the case of QCD above, for example, while up to 16 kinds of quark are permitted in the theory, it is currently believed that in fact only 6 are realized in nature. Since QCD could be consistently formulated even with only one type of quark, there is quite a bit of leeway in what is possible. As such, while the Goldilock's principle places a significant constraint on the kinds that can feature in an asymptotically free theory, this constraint fails to fully determine what those kinds are.

Now to repeat, what has been said on behalf of the constraints on kinds featuring in a fundamental theory applies only the asymptotically free theories - a very special class of fundamental QFTs. However, as was also underlined, we can expect there to be analogous (although not identical) constraints in the general case of asymptotically safe theories. As such, although

\footnotetext{
${ }^{44}$ Gross and Wilczek 1973.

${ }^{45}$ As Coleman later put it, "Theories of non-Abelian gauge fields and Fermi multiplets are sometimes asymptotically free and sometimes not. The fermions make a positive contribution to the beta-function; if the theory has too many fermions, the sign of beta is reversed and asymptotic freedom is lost" (Coleman 1985, p. 179).
} 
for ease of presentation we will take satisfaction of the Goldilock's principle to be a general constraint on fundamental theories, only something analogous to it will be true in general and that ought to be borne in mind. But for all that, what I want to claim now is that the existence of the GP (or its analogue) gives us reason for thinking that what fundamental kinds the world contains is not a brute and inexplicable fact about it. Rather, it is one for which we can give a partial explanation.

\section{Partially Explaining the Fundamental Kinds}

Recall that it is a tenet of physicalist brutalism that what fundamental kinds the world instantiates is a brute and inexplicable fact about it. As such, whatever it is that $\mathcal{F} \mathcal{K}$ turns out to be, it is not something for which any explanation can be given. This commitment we called $B(\mathcal{F} \mathcal{K})$.

Brutalism about fundamental kinds $(B(\mathcal{F} \mathcal{K}))$ : Suppose that, of the fundamental kinds, those instantiated in the world are $\mathrm{K}_{1}, \ldots, \mathrm{K}_{\mathrm{p}}$. Let these kinds be collectively denoted $\mathcal{F} \mathcal{K}$. Then that the fundamental kinds that are instantiated in the world are $\mathcal{F} \mathcal{K}$ is a fact for which no metaphysical explanation can be given.

If we want to argue against brutalism, then, we need to show that $B(\mathcal{F} \mathcal{K})$ is false - hence that at least some explanation can be given for the fact that the fundamental kinds instantiated in the world are the kinds $\mathcal{F} \mathcal{K}$. It must, however, be conceded at this point that it is unclear how to actually go about showing this, since the type of explanation that is relevant in this context is hardly immediately obvious. Given that $\mathcal{F} \mathcal{K}$ is a fundamental feature of the world, the fact of its existence is ex hypothesi ungrounded; and since $\mathcal{F} \mathcal{K}$ is not in any reasonable sense an 'event' it cannot be subject to causal explanation either. But since 'grounding explanation' is often taken as synonymous with 'metaphysical explanation', and 'causal explantion' as the paradigm of 'scientific explanation', it is wholly unclear what remaining options there are in the metaphysics of science for explaining what we want to explain. ${ }^{46}$

But our lack of familiarity with the relevant form of explanation might be just another expression of the brutalist bias. To argue that there is an explanation afoot here, I propose we adopt the following strategy. First, look for precedents in the literature on scientific explanation that have a relevantly similar formal structure to the explanation that would be appropriate here. Second, look to features pertaining to the content of that explanation to see if it may be characterized as 'metaphysical' in a recognizable sense. The reason I suggest proceeding in this way in particular, by looking to the philosophy of science for the relevant formal notions - is because only there does there seem to be any well-developed framework for modelling explanation in the absence of full determination. ${ }^{47}$ Since it is unclear why indeterministic explanation should

\footnotetext{
${ }^{46}$ I note that the other major notion of scientific explanation, namely unificationism, is regarded as the paradigm of 'explanation' in the sense of psychological achievement (see e.g. Craver 2014). I take it however that metaphysical explanation is necessarily ontic, and hence even if there were some explanation by unification available here I would not consider it a counterexample to brutalism. (Reasons for thinking - and rejecting - that $\mathcal{F} \mathcal{K}$ is partially grounded will be discussed below.)

${ }^{47}$ For example, Wilsch (2016, p. 15) states that 'Since there is no metaphysical analogue to statistical explanations, the laws of metaphysics are deterministic.' But no argument is given for this claim. Similarly Ruben (1992, p.
} 
be sanctioned in science and yet not in metaphysics (at least not without begging the question), should we succeed in locating a precedent in the philosophy of science with respect to the form of explanation we will be well on our way to undermining brutalism. ${ }^{48}$

Let's begin by mooting a 'general thesis about explanation' according to which 'we call for explanation only of what, although we know it is so, might have been otherwise for all else of some suitable sort we know'; that is, that we call for an explanation when there is a 'gap, between knowledge of what is and what must be'. ${ }^{49}$ Thus a proposed explanation will satisfy our demands to the extent that it succeeds in closing that gap - succeeds, that is, in ruling out other ways that the world might have been distinct from how it in fact is. It follows that, in the ideal case, the explanans will entail the explanandum. Schematic as it is, that observation surely captures something important about what we aspire to from explanations, and in the scientific context found its first expression in the deductive-nomological model proposed by Carl Hempel. ${ }^{50}$ This is a model intended to capture the scientific explanation of events, and amounts to the claim that we can be said to have explained an event when its occurrence may be deduced from premises expressing laws together with relevant statements of particular fact. As is well known, it did not take long for the shortcomings of the original DN-model to come into view (Hempel himself quickly abandoned it). But it was in any case obvious from the beginning that deductive entailment was untenable as a general demand on event explanation in science. Scientists, after all, have presumably been able to offer at least some explanation of all sorts of events even in cases in which they have been unable, perhaps unavoidably, to show that the explanandum follows with certainty given the explanans - whether it be a smoker contracting lung cancer or the successful detonation of a nuclear bomb. Thus models of explanation that relaxed the requirement of logical entailment, focussing instead on simply 'narrowing' the gap, began to be developed. Such explanations, given that they fail to determine the explanandum, we may dub 'indeterministic explanations'.

The examples just provided of smoking and uranium are canonical in the literature on indeterministic explanation, and more specifically canonical examples of probabilistic explanation. By now, of course, there exist a great many distinct proposals for how such explanation works. However, we need not concern ourselves with the vicissitudes of probabilistic explanation here, simply because no notion of probabilistic explanation seems appropriate for our purposes. The reason is simply that there doesn't seem to be anything in the considerations above that would

232): 'I do not think that there are any other nondeterminative explanations other than those which would arise on the basis of explanation by nondeterministic causes' - although he goes on to invite 'the reader [to] think of other candidates for nondeterministic relations that can be explanatory, other than nondeterministic causation'. This paper provides an example.

${ }^{48}$ It may be asked why I do not consider the counterfactual theory of explanation here - an approach to explanation that has been subject to a fair bit of contemporary discussion, partly on account of its seeming ability to cover both causal and non-causal explanations (see Reutlinger 2016). The reason is that the explanation purported here depends crucially on the assumption that fields are essentially, and hence necessarily, such as to evolve unitarily. But the counterfactual theory of explanation is inapplicable in cases in which the explanans is necessarily true (see Reutlinger op. cit., Section 4).

${ }^{49}$ Mellor op. cit., p. 234.

${ }^{50} \mathrm{Hempel}$ and Oppenheim 1948; Hempel 1965. If this seems a little staid, note that the 'papers [on the DN-model] and the reaction to them have structured subsequent discussion concerning scientific explanation to an extraordinary degree' (Woodward 2014). 
allow us to attach a probability, either absolute or relative, to $\mathcal{F} \mathcal{K}$ or any other inventory of fundamental kinds permitted by the $G P$ - hence nothing that would allow us to connect with any theory of probabilistic explanation at all.

Were probabilistic explanation the only form of indeterministic explanation the philosophy of science literature sanctioned, then, it would not furnish any resources for denying $B(\mathcal{F} \mathcal{K})$. Fortunately, however, and although rather less well-known, there is another notion lurking in the neighbourhood that is more congenial to our purposes. This is Hempel's notion of partial explanation. ${ }^{51}$ Another variant of the DN model, it holds that we have some claim to have an explanation of an event when we can deduce that some event in a circumscribed class will take place, without correspondingly being able to deduce that any particular member of that class is the one to be actualized.

Hempel illustrates what he has in mind with the following example (which I quote at length).

In his Psychopathology of Everyday Life, Freud offers the following explanation of a slip of the pen that occurred to him: "On a sheet of paper containing principally short daily notes of business interest I found, to my surprise, the incorrect date, "Thursday, October 20th," bracketed under the correct date of the month of September. It was not difficult to explain this anticipation as the expression of a wish. A few days before I had returned fresh from my vacation and felt ready for any amount of professional work, but as yet there were few patients. On my arrival I had found a letter from a patient announcing her arrival on the 20th of October. As I wrote the same date in September I may certainly have thought ' $\mathrm{X}$. ought to be here already; what a pity about that whole month,' and with this thought I pushed the current date a month ahead.

Clearly, the formulation of the intended explanation is at least incomplete [in that] it fails to mention any laws or theoretical principles in virtue of which the subconscious wish, and the other antecedent circumstances referred to, could be held to explain Freud's slip of the pen. However, the general theoretical considerations Freud presents here and elsewhere in his writings suggests strongly that his explanatory account relies on a hypothesis to the effect that when a person has a strong, though perhaps unconscious, desire, then if he commits a slip of pen, tongue, memory, or the like, the slip will take a form in which it expresses, and perhaps symbolically fulfills, the given desire. But clearly, such expression and fulfillment might have been achieved by many other kinds of slip of the pen than the one actually committed.

In other words, the explanans does not imply, and thus fully explain, that the particular slip, say $s$, which Freud committed on this occasion, would fall within the narrow class, say $W$, of acts which consist in writing the words "Thursday, October 20th"; rather, the explanans implies only that $s$ would fall into a wider class, say F, which includes $\mathrm{W}$ as a proper subclass, and which consists of all acts which would express and symbolically fulfill Freud's subconscious wish in some way or other.

The argument under consideration might be called a partial explanation: it provides complete, or conclusive, grounds for expecting $s$ to be a member of $F$, and since $W$ is a subclass of $F$, it thus shows that the explanandum, i.e., $s$ falling within $W$, accords with, or bears

\footnotetext{
${ }^{51}$ Note that Hempel's sense of 'partial explanation' differs from that of Ruben (op. cit., pp. 19-20). Ruben's sense seems to correspond to Hempel's 'elliptic explanation' (Hempel 1962, p. 16).
} 
out, what is to be expected in consideration of the explanans. By contrast, a deductivenomological explanation [...] might then be called complete since the explanans here does imply the explanandum. ${ }^{52}$

Although, like Hempel's original DN model, this variant of DN-explanation is concerned specifically with the explanation of events, from a formal point of view it mirrors precisely the situation we find ourselves in with respect to $\mathcal{F} \mathcal{K}{ }^{53}$ For whatever $\mathcal{F} \mathcal{K}$ is, we have "complete grounds for expecting' it to be a member of the 'wider class' of inventories of kinds that are compatible with the Goldilock's principle $G P$ - a constraint that rules out many, indeed infinitely many, combinations of kinds that might have been expected a priori. As such, this represents a non-trivial constraint on what $\mathcal{F} \mathcal{K}$ can be. However, we lack any resources at this point to go further and determine precisely which of the inventories within the class of those compatible with the GP is the particular one that is realized. For these reasons, structurally speaking we may be said to have a partial explanation for why the fundamental kinds that are instantiated in the world are the kinds $\mathcal{F} \mathcal{K}$.

Were Hempel correct in his initial intuition that the satisfaction of this schema gives us grounds for saying that we have a partial explanation, we would have grounds to stop at this point and declare brutalism to be false: for since the fundamental kinds instantiated by the world is now something for which partial explanation may be given, it is not the case that "no explanation' can be given of the fundamental after all. However, the idea that the satisfaction of the DN schema - and so also, we may take it, the weakened version foregrounded here - is sufficient for explanation came under attack almost as soon as the model itself emerged. As was forcefully pressed by Salmon and others, that idea would have as a consequence that we can explain the length of a flagpole by that of its shadow or the coming of a storm by the falling of a barometer. Observations such as these gave rise to a family of responses, each of which may be roughly but usefully surmised as holding that explanation consists of nomic expectability 'plus something else', such as the existence of underlying causal processes. ${ }^{54}$

This phenomenon that mere entailment does not suffice for explanation may also be witnessed in the context of metaphysical explanation - in particular, in the context of the literature on grounding explanation. In this context, it is universally held that while the claim that $\psi$ grounds $\phi$ implies that $\psi$ entails $\phi$, the converse is not true - grounding having been introduced into the literature explicitly as a relation more discerning than mere modal or logical notions. As such, mere entailment does not suffice for explanation even though a necessary feature it. ${ }^{55}$ For example, it is widely held that while one can claim that the existence of Socrates entails the

\footnotetext{
${ }^{52}$ Hempel 1962, pp. 17-18.

${ }^{53}$ One might want to object to this claim, on the grounds that a statement of law has not been included in the premises - only a statement concerning a structural feature of them (namely, that they must be unitary). But since we are not aiming at an explanation of events, this is entirely appropriate. In place of a law of nature we have a universally quantified claim - a 'metaphysical covering law' (cf. Sider (2011, p. 145) - about the nature of quantum fields, which we will argue below makes for a metaphysical explanation. (Note that Hempel himself (1965, p. 352) is explicit that DN-style explanations need not always be causal; see also Reutlinger 2016, Section 1. On the application of the DN model to metaphysical explanations, specifically grounding explanations, see Wilsch 2016.)

${ }^{54}$ See Woodward op. cit., Section 2.5 .

${ }^{55}$ See e.g. Rosen 2012, Section 5.
} 
existence of \{Socrates\} and also vice versa, only in the former case do we have a corresponding relation of grounding and hence an attendant explanation. However, just as in the case of scientific explanation, if one wants to claim that grounding ought be understood in terms of the satisfaction of formal relations plus something else, one needs to say something more about how that 'something else' is to be understood. What, then, can we say about what makes it the case that \{Socrates\}'s existence is grounded in that of Socrates, and not vice versa?

A widely-held answer is that the symmetry is broken in relations of grounding on account of the natures of the entities involved. That is, what accounts for the fact that \{Socrates\}'s existence is grounded in that of Socrates and not vice versa is some feature of the nature or essence of (at least one of) the relata. Such a view is explicit, for example, in the work of Fine, and also in that of Audi. ${ }^{56}$ As Audi puts it,

[G]rounding is sensitive to the natures of its relata... It is a necessary condition of one fact's grounding another that the natures of the properties involved be related to one another in some significant way. We can call this relation 'essential connectedness'...

We can characterize essential connection as follows: It lies in the nature of certain properties that their instances should stand in grounding relations. For example, when a given instance of maroonness grounds a coincident instance of redness, this fact manifests the natures of the relevant properties. It is part of their essence to behave in this way when instantiated. ${ }^{57}$

Thus to return to our example, we might say - with some plausibility - that it follows from the nature of \{Socrates\} that Socrates exists, and yet no way part of the nature of Socrates that impure sets of any kind exist. As such - and without pretending that every grounding theorist holds the same view - we may say that it is both widely held and intuitively plausible that grounding claims are backed by facts about natures, and hence that it is these that allow us to move (where we can) from the mere satisfaction of a formal schema to genuine instances of grounding explanation. ${ }^{58}$

In both scientific and metaphysical contexts, then, it is agreed that mere entailment relations must be 'backed' by some additional feature or property if genuine explanation is to ensue. Therefore whatever type of explanation might ultimately be involved in the explanation of the fundamental kind structure, the fact that we have satisfied the formal schema for a partial explanation is unlikely to establish that we have in fact partially explained it. Let us therefore focus now on the content of that putative explanation, and in order to do so it will help to recapitulate schematically the argument that has been laid out above.

1. The fundamental kinds are all kinds of quantum fields. (Stipulation as to the sort of entities populating the fundamental level.)

\footnotetext{
${ }^{56}$ See e.g. Fine 2012, p. 72; Audi 2012. (For Audi, the natures of both relata are involved; for Fine, only that of the grounded. But these differences do not matter for present purposes.) See also Rosen op. cit. for a more tentative version of the same view.

${ }^{57}$ Audi 2012, pp. 693-96.

${ }^{58}$ For example, Schaffer 2012 seems to take grounding connections to be primitive.
} 
2. Quantum fields are by their nature such as to evolve unitarily. (Stipulation as to the nature of quantum fields.)

3. The fundamental level is one in which the fundamental kinds $\mathcal{F} \mathcal{K}$ accord with fundamental laws. (Definition of 'fundamental level'.)

4. Fundamental laws are laws that are consistent in $E \rightarrow \infty$ limit. (Requirement on a fundamental law' for quantum fields.)

5. Fundamental laws of evolution must be unitary. (From 2 and 3)

6. Laws featuring a combination of kinds $K_{1}^{\prime}, \ldots, K_{r}^{\prime}$ not satisfying the Goldilock's principle not norm-preserving in the high-energy limit. (Demonstrated in Coleman and Gross 1973; Gross and Wilczek 1973).

C. $\therefore \mathcal{F} \mathcal{K}$ must belong to the class of combinations compatible with the Goldilock's principle (From 3, 4, 5, and 6).

As noted, this argument has the form of a partial explanation in Hempel's sense for why the fundamental kinds the world instantiates are the kinds $\mathcal{F} \mathcal{K}$ : for while we do not know precisely which combination of fundamental kinds will be realized, we know that whatever it is it will belong to a highly circumscribed class. Let us now therefore look for further hallmarks of genuine explanation that may be present in the premises, starting with premise 6. Presumably, the fact that laws featuring arbitrary combinations of fundamental kinds do not respect unitarity bounds, and hence are not unitary (since no longer norm-preserving) in the $E \rightarrow \infty$ limit, is a mathematical fact. To see this, note first that not every fact couched in mathematical language has a mathematical as opposed to, say, a causal explanation, because those mathematical facts could just be encoding functional relationships between certain physico-causal properties that are doing all the real explanatory work. ${ }^{59}$ Here, however, mathematics is employed in a sort of impossibility proof that shows there is no physical system that could exhibit the feature in question. As such, any explanation of the fact described in premise 6 cannot derive from features of extant physical stuff that happen to be described in mathematical language, but rather must issue directly from the relevant mathematical facts themselves. Furthermore, it is obvious that without this premise the argument would not be valid. Thus if - as has been defended - we take a mathematical explanation to be an explanation containing at least one mathematical element that is necessary to ensure the success of the explanation, then if our argument constitutes an explanation at all then it is, at least in part, a mathematical explanation. ${ }^{60}$

Turn now to premise 2. This premise consists of an explicit stipulation - one that I hope to have at least rendered defensible - as to the natures of the entities involved; as with premise 6, it is entirely unclear how one could reformulate the argument without it. Now, we already know that such an appeal to natures is (widely taken to be) that which transforms mere existential

\footnotetext{
${ }^{59}$ See Reutlinger 2014, Sec. 4.

${ }^{60}$ See Reutlinger ibid. for a defence of this criterion. I should say that mathematical explanation is a famously tricky notion, and it may be argued that the characterization here is too inclusive. But since my principal interest is in the metaphysical aspect of the purported explanation, I leave this issue for now.
} 
entailment into genuine grounding explanation. However, grounding seems not to be the type of explanation involved here. The reason is that it seems to be almost universally held that there cannot be partial grounding except as a component of full or deterministic grounding. Schaffer, for example, states outright that indeterministic grounding is an impossibility, and Fine, Audi, and Litland take a partial ground to be defined in terms of its being a component of a full ground (and not vice versa). ${ }^{61}$ Since there is no obvious way to promote our partial explanation into a deterministic explanation, if this is right then it follows that the fact of what fundamental kinds the world admits cannot be regarded as partially grounded. But if appeals to natures can convert mere entailment into explanation in the case of grounding, there is no reason to think that they cannot do explanatory work here. And while I do not claim to have a general account of 'metaphysical explanation' - indeed, I doubt that anyone does - the very mention of such language as 'natures' and 'essences' represents a warning bell for many that we have transgressed from science into metaphysics. ${ }^{62}$ Since the explanation here relies crucially on such appeals, I submit that it may properly be regarded as, at least in some essential part, a form of 'metaphysical explanation', its seeming distinctness from grounding notwithstanding. ${ }^{63}$

It seems, then, that our argument has not just the form of a partial explanation, but furthermore has content that carries the hallmarks of metaphysical and mathematical explanation. Since physics itself may be usefully thought of as an admixture of metaphysics and mathematics, it strikes me that this is precisely what we would expect of an explanation of the fundamental furnished by physics. As such, I think that we have good reason to say that what we have here is a genuine, if partial, explanation of why the world instantiates the fundamental kinds that it does in fact instantiate, and furthermore one that may properly be characterized as a metaphysical explanation, at least in some essential part. Since brutalism is the doctrine that the fundamental has no explanation, it seems to me that brutalism is no longer tenable. Rather, it appears that developments in science have allowed us to transcend this hallowed metaphysical doctrine, reducing - while of course not eliminating - that which we must simply take as brute. Since the illumination that comes by way of explanation is presumably a good, that strikes me as a conclusion worth celebrating. ${ }^{64}$

\footnotetext{
${ }^{61}$ See Schaffer 2016, p. 46 ; Fine 2012, p. 50; Audi 2012, p. 698; Litland op. cit., p. 20. We might argue for this insistence as follows: since the role of grounding in metaphysics is to organize the world into 'a tree-like structure of fundamentality' (Wilsch 2016, p. 1), and since something may be classed as non-fundamental only if it is fully determined by something else, there is no meaning we can attach to relations of grounding in the absence of full determination.

${ }^{62}$ See eg. Paul op cit., p. 5.

${ }^{63}$ Note however that my argument does not depend on the type of explanation involved here being distinct from (partial) grounding explanation. All that matters is that it can be regarded as sufficiently 'metaphysical' to represent a counterexample to brutalism - which a partial grounding explanation clearly would. (For an argument against the idea that partial grounds cannot occur in the absence of full, see Wilson 2014, pp. 543-44.)

${ }^{64}$ This conclusion is strongly evocative of Parfit's (1998b) discussion of attempts to explain why anything exists at all. There he notes that 'even if reality cannot be fully explained', there is nevertheless hope that 'we may still make progress' (p. 24) - although he cautions that 'if there is some explanation of the whole of reality, we should not expect this explanation to fit neatly into some familiar category' (p. 26) of scientific or distinctively philosophical explanation. Rather, we can expect to find ourselves in a situation in which 'there is no clear boundary here between philosophy and science', for at this level we are seeking explananda 'of the same kind as those that physicists are trying to discover' (p. 27). It will be clear that I have said essentially the same thing, mutatis mutandis, regarding the explanation of the fundamental.
} 


\section{Objections}

It must be admitted that the argument I have outlined above was rather involved - consisting as it did of many moving parts, and several contenious parts at that. As a result, I anticipate at least the following objections to be mounted against this denial of brutalism.

1. Mere partial explanation is not enough for metaphysical purposes. This objection cannot fully be assessed without deeper thought as to what purpose is served by explanation in metaphysics. But explanation is not an all-or-nothing affair in either science or everyday life, and I fail to see any reason to think that it ought to be that way in metaphysics. In the absence of some further defence of this claim, it simply begs the question.

2. The argument only shows that our assumption that $\mathcal{F} \mathcal{K}$ is a fundamental feature of the world was incorrect. Given that $\mathcal{F} \mathcal{K}$ is not fully determined, this cannot be right on any determination-based conception of the relation between the fundamental and nonfundamental, such as those based on grounding. (Without full determination, the nonfundamental loses its 'free lunch' character.) And were we to adopt a different conception of fundamentality, such as the 'joint-carving' notion of Sider, then I can only repeat that the fundamental kinds have always been taken as paradigms of joint-carving notions. As such, resorting to this response at best implants us in the thick of Duhem-Quine territory. But the refusal to give up brutalism whatever the cost would render it more a regulative principle than a substantive metaphysical claim, and it should be asked what it is about brutalism that really entitles it to such protected status.

3. Since it proceeds from an overtly non-Humean assumption that fields are essentially such as to evolve unitarily, the conclusion is trivial. Again, there is a lot to say here. But for now, suffice to say that the explanation offered here of why the fundamental kinds instantiated in the world are the kinds that they are is importantly disanalogous from the sort of anti-Humean explanations offered by, for example, dispositional essentialists. What dispositional essentialists claim to have an explanation for is the regularities we observe in the causal order of events, and what are posited in order to explain them are 'dispositional essences' that are explicitly designed to reproduce such regularities. As such, one can certainly argue that the resulting essence-based explanations for observed regularities are unilluminating and trivial. Here, however, we start with a stipulation regarding the nature of quantum fields of any kind - that they are the sort of thing to evolve unitarily - and end up with a partial explanation of what the actual fundamental kinds are. But to be vulnerable to the objection that the essence-based explanation offered here is as trivial as that issuing from dispositional essentialism, we would have to be claiming to have an explanation not of what the actual fundamental kinds are, but rather of (something like) why the probabilities associated with determinate measurement outcomes are given by the Born rule. But we - while surely being committed to it - make no claim to having explained that. What we do claim to have an explanation for is a fact distinct in almost every respect from that which was put in by hand, and as such I do not see how it can be regarded as 'trivial' in anything like the above sense. ${ }^{65}$

\footnotetext{
${ }^{65}$ I note further that nowhere, to my knowledge, in the literature on dispositional essentialism does anyone claim to
} 
There is much more to be said about both the content of these objections and the responses to them; no doubt numerous other objections wait in the wings too. But I hope at least to have shown that brutalism, if it is to remain axiomatic in metaphysics, stands in need of a far more developed articulation and defence that it has received until now. To those who continue to feel, despite all my protestations, that the explanation offered here somehow fails to qualify as sufficiently 'metaphysical' to falsify brutalism, perhaps they can take the opportunity to explain why such a view is forced upon us, or what its benefits are supposed to be. For such a situation cannot help but remind me of similar protests to the effect that scientific explanations are in principle incapable of falsifying religious doctrines either. So if nothing else, let this be an invitation for brutalists to outline more precisely the content of their hypothesis and its felt motivations, and what it is about physics that in principle cannot surmount the explanatory gap that has been taken to define the central subject matter of metaphysics.

\section{Acknowledgments}

I am very grateful to Reuven Brandt, Steven French, Nick Huggett, Joseph Stratmann, Elanor Taylor, the UCSD philosophy of physics reading group, three anonymous referees, and an audience at UIC / Geneva for their indispensible comments and criticisms.

\section{References}

- Armstrong, David M. (1997) A world of states of affairs, Cambridge University Press.

- Árnad'ottir, Steinvör Thöll and Tim Crane (2013). 'There is no exclusion problem.' In Sophie C. Gibb and Rögnvaldur Ingthorsson (eds.), Mental Causation and Ontology. Oxford University Press.

- Audi, Paul (2012). 'Grounding: Toward a Theory of the In-Virtue-Of Relation'. Journal of Philosophy 109 (12):685-711.

- Barnes, Eric (1994). 'Explaining Brute Facts'. In PSA: Proceedings of the Biennial Meeting of the Philosophy of Science Association, Vol. 1994, Volume One: Contributed Papers (1994), pp. 61-68.

- Cameron, Ross P. (2015). The Moving Spotlight: An Essay on Time and Ontology, Oxford University Press.

- Castellani, Elena (2002). 'Reductionism, Emergence, and Effective Field Theories'. Studies In History and Philosophy of Science Part B: Studies in History and Philosophy of Modern Physics 33 (2), pp. 251-267.

- Chakarvartty, Anjan (2007). A Metaphysics for Scientific Realism: Knowing the Unobservable. Cambridge University Press.

- Coleman, Sidney (1985). Aspects of Symmetry: Selected Erice Lectures, Cambridge University Press

have an explanation of the identities of the fundamental natural kinds, as opposed to aspects of their behaviour. Thus we are here explaining some of what the dispositional essentialists take as brute (see e.g. Chakravartty $o p$. cit. again). 
- Coleman, Sidney and David J. Gross (1973). 'Price of Asymptotic Freedom'. Phys. Rev. Lett. 31 (Sept 1973), pp. 851-4.

- Collins, John (2008) Renormalization: An Introduction to Renormalization, the Renormalization Group and the Operator-product Expansion, Cambridge University Press.

- Craver, C. (2014). 'The ontic account of scientific explanation'. In M. I. Kaiser, O. R. Scholz, D. Plenge, and A. Hütteman (Eds.), Explanation in the special sciences: The case of biology and history, Synthese Library 367. Dordecht: Springer.

- Dasgupta, Shamik (2016). 'Metaphysical Rationalism'. Noûs 50 (2):379-418.

- deRosset, Louis (2010). 'Getting priority straight'. Philosophical Studies 149 (1):73-97.

- Fahrbach, Ludwig (2005). 'Understanding Brute Facts'. Synthese 145: 449466.

- Fine, Kit (1995). 'Ontological Dependence'. Proceedings of the Aristotelian Society 95: 269 290.

- Fine, Kit (2012). 'Guide to Ground'. In Fabrice Correia and Benjamin Schnieder (eds.), Metaphysical Grounding. Cambridge University Press 37-80.

- Gross, David J. and Frank Wilczek (1973). 'Ultraviolet Behaviour of Non-Abelian Gauge Theories'. Phys. Rev. Lett. 30 (26), pp. 1343-1346, Nov. 1973.

- Hall, Ned (2010). 'David Lewis's Metaphysics'. The Stanford Encyclopedia of Philosophy (Fall 2010 Edition), ed. E. N. Zalta.

- Hempel, Carl (1962). 'Explanation in Science and History'. In R.G. Colodny (ed), Frontiers of Science and Philosophy, Pittsburgh: The University of Pittsburgh Press.

- Hempel, Carl, (1965). Aspects of Scientific Explanation and Other Essays in the Philosophy of Science, New York: Free Press.

- Hempel, Carl and Paul Oppenheim (1948). 'Studies in the Logic of Explanation'. Philosophy of Science, 15: 135175. Reprinted in Hempel 1965, pp. 245290

- Hospers, John (1997). An Introduction to Philosophical Analysis, Pearson.

- Hudson, Hud (1997). 'Brute Facts.' Australasian Journal of Philosophy, 75 (1), March 1997, pp. 77-82.

- Huggett, Nick and Robert Weingard (1995). 'The renormalisation group and effective field theories'. Synthese 102 (1): 171 - 194.

- Ismael, J. and Schaffer, J. (forthcoming) 'Quantum Holism: Nonseparability as Common Ground'. Synthese.

- Litland, Jon Erling (2013). 'On Some Counterexamples to the Transitivity of Grounding'. Essays in Philosophy 14 (1):3.

- Loewer, Barry (2001). 'From Physics to Physicalism'. In Physicalism and its Discontents, eds. Carl Gillett and Barry Loewer (pp. 39-56), Cambridge: Cambridge University Press.

- Loewer, Barry (2012). 'Two Accounts of Laws and Time'. Philosophical Studies 160 (1):115-137.

- Mellor, D. H. (1976). 'Probable explanation'. Australasian Journal of Philosophy 54 (3):231-241.

- Melnyk, Andrew (1997). 'How to Keep the "Physical” in Physicalism'. The Journal of Philosophy, Vol. 94, No. 12 (Dec., 1997), pp. 622-37. 
- Miller, Kristie (2009): 'Defending Contingentism in Metaphysics'. dialectica Vol. 63, No. 1, pp. 23-49.

- Myrvold, Wayne C. (2015). 'What is a wavefunction?'. Synthese 192 (10): 3247-74.

- Parfit, Derek (1998a). 'Why Anything? Why This?'. London Review of Books, Vol. 20 No. 2: 22 January 1998, pp. 24-27.

- Parfit, Derek (1998b). 'Why Anything? Why This?' (cont'd). London Review of Books, Vol. 20 No. 8: 16 April 1998, pp. 24-27.

- Paul, L. A. (2012). 'Metaphysics as modeling: the handmaiden's tale'. Philosophical Studies 160 (1): $1-29$.

- Percacci, R. (2009). The asymptotic safety FAQs. Webpage, URL http://www.percacci.it/roberto/physics/as/faq.html

- Reutlinger, Alexander (2014). 'Why Is There Universal Macrobehavior? Renormalization Group Explanation as Noncausal Explanation'. Philosophy of Science 81 (5):1157-1170.

- Reutlinger, Alexander (2016). 'Does the Counterfactual Theory of Explanation Apply to NonCausal Explanations in Metaphysics?'. European Journal for Philosophy of Science: 1-18.

- Rosen, Gideon (2010). 'Metaphysical Dependence: Grounding and Reduction'. In Bob Hale and Aviv Hoffmann (eds.), Modality: Metaphysics, Logic, and Epistemology. Oxford University Press 109-36.

- Ruben, David-Hillel (1992). Explaining Explanation, Routledge: London.

- Saatsi, Juha and Alexander Reutlinger (forthcoming). Explanation Beyond Causation. Oxford University Press.

- Salmon, W. C. (1989). Four decades of scientific explanation. Pittsburgh: University of Pittsburgh Press.

- Schaffer, Jonathan (2003). 'Is There a Fundamental Level?', Nous, Vol. 37, No. 3, pp. 498-517.

- Schaffer, Jonathan (2009). 'On what grounds what'. In David Manley, David J. Chalmers and Ryan Wasserman (eds.), Metametaphysics: New Essays on the Foundations of Ontology. Oxford University Press 347-383.

- Schaffer, Jonathan (2010). 'Monism: The Priority of the Whole'. Philosophical Review 119 (1):31-76.

- Schaffer, Jonathan (2016). 'Grounding in the image of causation'. Philosophical Studies 173 (1):49-100.

- Sider, Theodore (2009). 'Ontological realism'. In David John Chalmers, David Manley and Ryan Wasserman (eds.), Metametaphysics: New Essays on the Foundations of Ontology, Oxford University Press.

- Sider, Theodore (2013). 'Against Parthood'. Oxford Studies in Metaphysics 8:237-293.

- Strawson, Galen (2006). 'Realistic monism - why physicalism entails panpsychism'. Journal of Consciousness Studies 13 (10-11): 3-31.

- Wallace, David and Christopher G. Timpson (2010). 'Quantum Mechanics on Spacetime I: Spacetime State Realism', The British Journal for the Philosophy of Science, Vol. 61, No. 4, pp. 697-727. 
- Weinberg, Steven (1979). 'Ultraviolet divergences in quantum theories of gravitation. In General Relativity: An Einstein Centenary Survey, eds. S. W. Hawking and W. Israel (pp. 790-831), Cambridge University Press.

- Weinberg, Steven (1994). Dreams of a Final Theory (2nd edition), Vintage.

- Wilsch, Tobias (2016). 'The Deductive-Nomological Account of Metaphysical Explanation'. Australasian Journal of Philosophy 94 (1):1-23.

- Wilson, Jessica M. (2014). 'No work for a theory of Grounding'. Inquiry 57 (5-6):535-579.

- Woodward, James, 'Scientific Explanation', The Stanford Encyclopedia of Philosophy (Winter 2014 Edition), Edward N. Zalta (ed.). 